felt perfectly well. On the most careful examination I could find nothing else.

20th. - The same; but the boy could not understand being kept in bed when he felt perfectly well, and I could not sanction his getting up while his temperature and pulse were above normal.

21st.-After another careful and minute examination, owing to his temperature and pulse remaining the same, $\mathbf{I}$ detected a very soft and faint mitral systolic murmur.

This gradually increased in intensity every day, and on the 24th his temperature became normal, and continued so, but his pulse remained high for a time. He was kept quietly in bed for another ten days.

I carefully talked over the illness with his father, and told him he had endocarditis, which was a very serious matter; though by dint of the very greatest care, in not allowing any exertion for the next few months, its effects might all disappear ; otherwise he would carry a maimed heart for the rest of his days.

On March 8th he was unfortunately suddenly told he might go home for the rest of the term. Next morning when I saw him I was informed that he had been in a curious state, talking incoherently about things that had no existence at all, and he seemed thoroughly dazed at my visit. He was cheerful and happy, though incoherent, and had a quiet pulse. Whether his joy was too much for his state, or whether minute particles of fibrine were liberated from the mitral valve in the excitement of his sudden joy at the idea of going home, I cannot say; but I am inclined to think that this latter is what happened.

On June 11th I auscultated his heart, and there was not the slightest trace of murmur remaining. He was quite well.

The lessons to be learnt from the case, I think, are1. That the most trivial amount of rheumatism may be accompanied by endocarditis. 2. That recent murmurs from endocarditis may and do disappear in young healthy subjects by dint of very great care while they are recent. Rugby.

\section{A CASE OF SUDDEN DEATH DURING ETHER ADMINISTRATION.}

\section{BY ROBERT N. HARTLEY, M.R.C.S.}

THE rarity of cases of sudden death during the use of ether as an anæsthetic is my excuse for entering somewhat fully into the following details.

The patient was a gentleman, aged sixty-six, who had for some weeks previously been the subject of symptoms which eventually culminated in complete obstruction of the bowels, the site of the obstruction being thought to be either in the lower part of the descending colon or the sigmoid flexure.

As the only procedure holding out any hope of prolonging life, it was decided, in accordance with the opinion of Sir James Paget and Mr. Pridgin Teale, and with the full consent of the patient, to open the bowel in the right lumbar region.

Before the commencement of the operation the patient was lying, or half-lying, in bed, with the head and shoulders well propped up with pillows, breathing regularly and with. out great effort. There was no complicating disease in heart or lungs, and the pulse, though rather quick and small, was not inordinately feeble. There was thus nothing to contraindicate the use of an anæsthetic, or to make one more than usually anxious as to its effect. Some artificial teeth worn by the patient had been previously taken out, and one of the pillows was now removed for the more easy adjustment of the inhaler.

Ether was administered by Clover's smaller inhaler, two ounces being the quantity contained in the ether-chamber. The patient took the ether without the slightest hesitation or resistance, moderately inflating the india-rubber bag with each expiration, and in two minutes was quite anæsthetic He was now placed on his left side with the head lower, and in five minutes from the commencement of the administration of ether the line of incision was made by Mr. Teale. The patient was never allowed to become deeply "carbonised," and the inhaler was frequently withdrawn entirely. In ten minutes from the commencement of the operation, and just as I was engaged in counting the carotid pulse, which was beating evenly and with a rather stronger impulse that before the operation, he showed an inclination to vomit, and ejected a quantity of brownish fluid smelling strongly u: brandy. He then took one deep inspiration, and seemed is if inclined to vomit again; but his head sank back on the pillow, and he quietly died, making no further respiratory effort, except one short gasp during the performance of arti. ficial respiration. Prompt exploration of the fauces failci. to show any mechanical obstruction to the breathing, and in spite of an immediate resort to artificial respiration and al' other available means for restoring animation, our efiurt: proved useless. An examination of the inhaler showed that a little more than an ounce of ether had been spent.

No post-mortem examination was made.

The interest of this case centres in the difficulty of de. fining the exact cause of death. The mode of death is one which unfortunately is not rare during the use of chlorofur' as an anæsthetic, but is almost unknown while a patient is under ether.

There was no mechanical obstacle to respiration by the regurgitation of anything into the larynx during the act vi vomiting. Nothing solid had been introduced into the stomach for seven or eight days previous to the operation, and the vomited material consisted entirely of thin fluid. Moreover, there was no paroxysmal or convulsive seizure such as would be likely to follow a sudden mechanical in. terference with the act of breathing.

Two circumstances may have contributed to the suddenly fatal result :-

First, the distension of the bowels possibly interfering to some extent with the descent of the diaphragm, and hence with easy respiration, though neither before nor during the administration of ether had the patient shown any con. siderable difficulty or distress of breathing. Mr. Teale regrets, however, that he did not at once complete the operation, and rapidly open the bowel, and thereby reduce one factor-the distension by gas.

Secondly, the undoubted tendency to death from asthenia in cases of abdominal obstruction. In this particular case, the lungs having been more or less emptied of air by the act of vomiting, the additional quantity of blood drawn into the heart and large vessels by the next inspiratory effort might easily have brought the feebly acting heart to a stand. still. Mr. Clover suggests to me that by the exuctation of fluid containing brandy the ether may have been mixed with alcoholic vapour, which would add to its narcotising effect, and thereby tend to increase the cardiac depression.

Brixton-road, S.W.

\section{ABSCESS OF THE CEREBELLUM.}

BY W. A. BERRIDGE, M.R.C.S., L.S.A.

I TRUST a general practitioner may be allowed to put upon record the following brief notes of a case of abscess of the cerebellum, as a small token of his gratitude for the instruction he received from Dr. J. Hughlings Jackson, F.R.S., and for the care bestowed upon his clinical education by his former teacher and now esteemed friend, Dr. Stephen Mackenzie.

A. D—, aged seventeen, had suffered from time to time since he was two years old from "gatherings in his head," which "broke," and "smelled very bad." He went to school when five years old, but never got on well. The schoolmaster said "he was stupid, and would not learn." Still he learned his letters, and could hold his own in play with other boys. He had no particular illness, never had scarlet fever, and had no signs of congenital syphilis, but often suffered from headaches and the aforesaid "gatherings."

When first seen (August, 1879) he was tall, pale, and rather thin. He spoke as I have noticed patients with oromic cerebral mischief generally do, his words beginning with a "jerk" and ending with a drawl; or, in other words, the first part of a word being sharp and rather acute, the latter part grave and always prolonged. His mother said, "I have sent for you because he has a cold in his head, and cannot smell." On further inquiry, " he had headaches, occasional retchings, and felt giddy"; but he could not describe his "giddiness." He had no "reel." He ate ravenously. Urine 10lö, acid. no albumen, no sugar. Temperature normal. He had no para. lysis. Pupils dilated, butr esponded to light. 